\title{
On Edwards' Model for Polymer Chains III. Borel Summability
}

\author{
John Westwater \\ Department of Mathematics, University of Washington, Seattle, WA 98195, USA
}

\begin{abstract}
The basic existence theory for Edwards' model of long polymer chains is completed.
\end{abstract}

\section{Introduction}

In [1] we proved an existence theorem for a probability measure on continuous paths in space, proposed by Edwards [2] as a stochastic model for the geometric properties of long polymer chains. This theorem was limited to sufficiently small values of the coupling constant $g$, and, as noted in the introduction to [3], this restriction is highly unsatisfactory, since the most interesting question concerning the Edwards model is a question about its asymptotic behavior in the limit $g \rightarrow \infty$. In the present paper we show that the polymer measure $v(g)$ is well defined for all $g \geqq 0$. In addition we show that the (renormalised) perturbation series for moments of $v(g)$, although (presumably) divergent, determine the moments as their Borel sums. This result is of interest because it shows that all information about the model is implicitly contained in the perturbation series, and hence guarantees the uniqueness of our construction of the model. In a final section we confirm the expectation of Symanzik [4], that the measure $v(g), g>0$, and the Wiener measure $\mu=v(0)$ are mutually singular, by showing that $v\left(g_{1}\right)$ and $v\left(g_{2}\right)$ are disjoint for any $g_{1}, g_{2}$ with $0 \leqq g_{1}<g_{2}$.

Throughout this paper we rely heavily on the results of [1]. In particular the notation of [1] will be used without further explanation.

\section{Borel Summability}

We adopt the notation and hypotheses of Theorem 1 [1], and consider, for a given localised random variable $R \in L^{q}(\Omega, G(m), \mu)$, for some $m \geqq 0, q>1$, the expectation

$$
\begin{aligned}
E_{v(g)}[R] & =E\left[R f_{m}(g)\right] \\
& =\lim _{n \rightarrow \infty} \frac{E[R \exp [-g S(n)]]}{E[\exp [-g S(n)]]} \\
& =F(R, g)[F(1, g)]^{-1},
\end{aligned}
$$


with

$$
F(R, g)=\lim _{n \rightarrow \infty} E[R \exp [-g S(n)]] C_{n}(g)^{-1} .
$$

According to $[1], F(R, g)$ is well-defined for $g$ positive, and sufficiently small. In this section we will prove

Theorem 1. For some $g_{0}>0, F(R, g)$ is well-defined for all $g \in D=\{g: \operatorname{Reg}>0$ $\left.|g|<g_{0}\right\}$, and is bounded and holomorphic in $D$. As $g \rightarrow 0$ in $D, F(R, g)$ admits an asymptotic expansion

$$
F(R, g) \sim \sum a_{l}(R) g^{l} .
$$

If, in addition, the constants $\beta_{1}, \beta_{2}$ appearing in conditions $C 4, C 5$ of [1] satisfy $\beta_{1}, \beta_{2}<2$, then $F(R, g)$ is the Borel sum of its asymptotic expansion.

Remark. For the Edwards' model $\beta_{1}=\frac{3}{2}$, and $\beta_{2}=\frac{3}{2}+\varepsilon$ with an $\varepsilon>0$, which may be made arbitrarily small by choice of the Hölder index $q$ which appears in the verification of $C 5$ ([1] p. 169). Thus Theorem 1 implies Borel summability for the Edwards' model.

Proof. We begin the proof of Theorem 1 with a lemma which makes explicit the dependence of the majorant for the inductive expansion on the choice of termination sequence $J$. For $L>0$ an arbitrary integer, denote by $J_{L}$ the inductive expansion defined by the termination sequence $j(m, n)=\left[L \rho^{n+m}\right]$, with $\rho>1$ chosen as in [1] 2.7. Let $B$ denote the truncation sequence $b_{n}=K \xi^{n}$, with $\xi$ chosen so $1<\xi<\min$ $\left(2^{1 / 2}, \rho^{1 / 2}\right)$, and $K>0$ arbitrary. Write $\beta=\max \left(\beta_{1}, \beta_{2}, 1\right)$, and choose $\gamma>\beta-1$. Then we have

Lemma 1. Let $R$ be a localized random variable, with $R \in L^{q}$ for some $q>1$, and $r=\max \{n: n \in \operatorname{loc}(R)\}$. Then there exist $\varepsilon>0$, and $M>0$, such that, for all $L \geqq 1$, and $g$ satisfying $|g| \leqq 1$

$$
J_{L}\{F(g, R ; B)\} \prec M\|R\|_{q}\left(1-\varepsilon|g| L^{\gamma}\right)^{-(r+1)} .
$$

Proof of Lemma 1. The proof is a recapitulation of the majorisation argument of [1] 2.7 with certain changes which are needed to give the correct dependence of the estimates on $L$.

The truncation sequence $B$ is chosen more rapidly rising than in [1] in order to simplify the subsequent passage from $F(g, R ; B)$ to $F(g, R)$. The condition $\xi<2^{1 / 2}$ implies $b_{n+k} \theta^{-k} \leqq b_{n}$ for all $n, k \geqq 0$ with $\theta=2^{1 / 2}$ (cf. [1] 2.7.2, and the argument following 2.7.37); $\xi<\rho^{1 / 2}$ guarantees [1] 2.7.21.

The introduction of complex $g$ entails a minor modification in the estimation of the termination factors $P_{n}^{2}$; thus [1] 2.7 .14 is to be replaced by

$$
\begin{aligned}
\|\exp \{-g t(n, m) Z(n, m ; B)\}\|_{\boldsymbol{r}(n)}^{\boldsymbol{r}(n)}= & \|\exp \{-(\operatorname{Reg}) t(n, m) X(n, m ; B)\}\|_{\boldsymbol{r}(n)}^{\boldsymbol{r}(n)} \\
& \cdot\|\exp \{(\operatorname{Im} g) t(n, m) \Gamma(X(n, m ; B))\}\|_{r(n)}^{r(n)}
\end{aligned}
$$

with $r(n)=p p(n) c(n)$. The first factor is to be estimated as in [1] (note that the sign condition on $g$ in [1] Lemma 7 is irrelevant, as is clear in [1] 2.7.16). The second factor is a Gaussian integral equal to

$$
\begin{aligned}
E\left[\exp \left\{\frac{1}{2}|\operatorname{Im} g|^{2} t(n, m)^{2} E\left[X(n, m ; B)^{2}\right] r(n)^{2}\right\}\right] & \leqq \exp \left\{\frac{1}{2}|g|^{2} E\left[X^{2}\right] r(n)^{2}\right\} \\
& \leqq \exp \left\{A r(n)^{2}\right\},
\end{aligned}
$$


for $|g| \leqq 1$, with $A$ some constant. This is of the same form as the bound obtained in [1] for the first factor, so the form of the bound obtained for (5) is unchanged, and the argument of [1] can proceed.

We come now to the changes in [1] 2.7 needed to give the correct dependence on $L$ of the majorant; this dependence enters through the constant $C_{13}$ of [1] 2.5.28 - to prove Lemma 1 it clearly suffices to show $C_{13}=O\left(L^{\gamma}\right)$, or, equivalently (see [1] 2.5) $K_{4}=O\left(L^{\gamma}\right)$ and $K_{8}=O\left(L^{\gamma}\right)$. First note that in the argument following [1] 2.7.32 the essential property of the comparison random variables $Y$ is that $E[\exp (x|Y|)]$ exist for sufficiently small $x .{ }^{1}$ Thus at this step we can use exponential rather that Gaussian random variables, which makes it possible to replace $p^{1 / 2}$ by $p$ in [1] 2.5.28. We make this change, and the corresponding change in [1] 2.5.21, 2.5.25, and claim that we can then obtain $K_{4}=O\left(L^{\gamma}\right), K_{8}=O\left(L^{\gamma}\right)$. To do this we require a further lemma; the first part of the lemma replaces [1] Lemma 3(a) and the second sharpens [1] Lemma 5. The proof of the lemma is a minor modification of the proofs of the lemmas in [1] which it replaces; we omit it.

Lemma 2. Let $X$ be a random variable with $E[X]=0, N$ a positive integer, $X_{1}, \ldots, X_{N}$ independent random variables with the same distribution as $X$, with normalised sum

$$
S_{N}=N^{-1 / 2}\left[X_{1}+\ldots+X_{N}\right]
$$

Suppose that for some $C>0, \beta \geqq 1$, and all $p \geqq 1$,

$$
\|X\|_{p} \leqq C p^{\beta} \text {. }
$$

Then (a) for any $\varepsilon>0$, there exists $\alpha>0$ and $K>0$ such that for any $A \geqq 1$

$$
\left\|S_{N}\right\|_{p} \leqq K A^{\beta-1+\varepsilon} p
$$

for all $N \geqq 1, p \leqq A N^{\alpha}$.

(b) There exists $K>0$ such that for all $N \geqq 1, p \geqq 1$,

$$
\left\|S_{N}\right\|_{p} \leqq K p^{\beta} \text {. }
$$

The use of Lemma 2 in the argument of [1] gives $K_{4}=O\left(L^{\gamma}\right)$. To obtain $K_{8}=O\left(L^{\gamma}\right)$ requires a further change in the argument of [1], since the constant $\beta_{8}$ given by [1] Lemma 4 is not satisfactory. Instead we use [1] Lemma 4 only for $p=2$ to transfer $C 6^{\prime}$ to the truncated array, and then repeat for the truncated array the argument that $C 6^{\prime}$ implies $C 6$ ([1] 3.3 Lemma 3), using the above Lemma 2(b) in place of [1] Lemma 5. This yields [1] 2.5.11 with the constant $\beta_{8}=\beta+\varepsilon$, for any given $\varepsilon>0$. Then the argument of [1] gives [1] 2.5.25 (modified by the replacement of $p^{1 / 2}$ by $p$ on the right side) with $K_{8}=O\left(L^{\gamma}\right)$, which completes the proof of Lemma 1.

The next lemma casts the basic estimate implying Borel summability into a

1 Here we take the opportunity to correct an error in [1]. We should have introduced independent Gaussian variables $Y(n, m), n \geqq 0, m \geqq 1$. Then [1] 21.34 holds with $H(x)=\prod^{\infty} K\left(x \tau_{17}^{m}\right), K(x)=$ $E[\exp [x|Y|]]$, and the argument proceeds as in $[1]$ 
convenient form for use with our majorisation arguments. We omit the straightforward proof.

Lemma 3. For $\varepsilon>0$ write $D(\varepsilon)=\{g: \operatorname{Reg}>0,|g|<\varepsilon\}$. Suppose $F(g)$ bounded and holomorphic in $D(\varepsilon)$ for some $\varepsilon>0$, and $\gamma \geqq 0$. Then the following conditions on $F$ are equivalent

(a) As $g \rightarrow 0$ in $D(\varepsilon), F(g)$ admits an asymptotic expansion

$$
F(g) \sim \sum a_{j} g^{j}
$$

for which

$$
\left|F(g)-\sum_{j=0}^{L-1} a_{j} g^{j}\right| \leqq C^{L}|g|^{L} L^{\nu^{L}}
$$

for some $C>0$, all $L \geqq 1$ and $g \in D(\varepsilon)$.

(b) There exists a $C>0$ such that for each $L \geqq 1$, and $g \in D\left(C^{-1} L^{-\gamma}\right), F(g)$ admits an expansion

$$
F(g)=\sum_{k=0}^{\infty} F_{k, L}(g)
$$

such that

(i) each term $F_{k, L}(g)$ is holomorphic in $g$ in $D\left(C^{-1} L^{-\gamma}\right)$, and satisfies a bound

$$
\left|F_{k, L}(g)\right| \leqq|g|^{w(k, L)} B_{k, L}
$$

with $w(k, L)$ an integer called the weight of the term, and $B_{k, L} \geqq 0$,

$$
\sum_{k: w(k, L)=w} B_{k, L} \leqq C\left(C L^{\gamma}\right)^{w}
$$

(iii) if $w(k, L)<L$, then $F_{k, L}(g)$ is a monomial in $g$.

Remark 1. (b) implies $F$ bounded and holomorphic in $D(\varepsilon)$ with $\varepsilon=C^{-1}$.

Remark 2. (ii) is equivalent to the existence of a majorisation

$$
\sum B_{k, L}|g|^{k}<h\left(|g| L^{\gamma}\right),
$$

with $h(z)=\sum h_{n} z^{n}, h_{n} \geqq 0$ for all $n$, holomorphic in the neighbourhood of $z=0$.

Remark 3. According to a theorem of Nevanlinna (see Sokal [5]), if $\gamma \leqq 1$ (a) implies that the asymptotic series for $F$ is Borel summable to $F$; if $\gamma<1$ the Borel transform is an entire function.

We have

with

$$
F(R, g)=F_{1}(g ; B) F_{2}(R, g ; B)
$$

$$
F_{1}(g ; B)=\lim _{n \rightarrow \infty} C_{n}(g ; B) C_{n}(g)^{-1},
$$

an entire function of the form $\exp \left\{a g+b g^{2}\right\}$ ([1] Lemma 2), and

$$
F_{2}(R, g ; B)=\lim _{n \rightarrow \infty} E[R \exp [-g S(n)]] C_{n}(g ; B)^{-1}
$$


Thus the proof of Theorem 1 will be complete if we show that $F(g)=F_{2}(R, g ; B)$ satisfies condition (b) of Lemma 3.

Write

$$
T=\sum_{n=0}^{\infty}[X(n)-X(n ; B)]
$$

and, for $m \geqq 0$,

$$
T_{m}=E[T \mid G(m)]
$$

With the truncation sequence $B$ specified in Lemma 1 , it is easy to check

Lemma 4. There exist constants $C>0$, and $\tau<1$, such that, for all $p \geqq 1, m \geqq 0$,

$$
\begin{aligned}
& \|T\|_{p} \leqq C p^{1+\gamma}, \\
& \|T-E[T \mid G(m)]\|_{p} \leqq C \tau^{m} p^{1+\gamma} .
\end{aligned}
$$

For any integer $L>0$, define functions $e_{L, j}(u)$ of a real variable $u$ for integral $j \geqq 0$

$$
\begin{aligned}
e_{L, j}(u) & =(-1)^{j} \frac{u^{j}}{j !}, \quad 0 \leqq j<L, \\
e_{L, j}(u) & =\exp \{-u\}-\sum_{j=0}^{L-1} e_{L, j}(u), \quad j=L, \\
e_{L, j}(u) & =0, \quad j>L,
\end{aligned}
$$

so that $\exp \{-u\}=\sum_{j=0}^{\infty} e_{L, j}(u)$ is the expansion of $\exp \{-u\}$ as a Taylor polynomial of degree $L-1$ plus a remainder.

Denote by $G(-1)$ the trivial $\sigma$-algebra, so that $T_{-1}=E[T \mid G(-1)]=E[T]$. We will show that the expansions

$$
\begin{aligned}
F_{2}(R, g ; B)= & \left\{\sum_{j=0}^{\infty} \frac{(-g E[T])^{j}}{j !}\right\} J_{L}\{F(g, R)\} \\
& +\sum_{m=0}^{\infty} \sum_{j=0}^{\infty} J_{L}\left\{F\left(g, R\left[e_{L, j}\left(g T_{m}\right)-e_{L, j}\left(g T_{m-1}\right)\right]\right)\right\}
\end{aligned}
$$

satisfy (b) of Lemma 3.

(bi), (biii) are evidently satisfied, the weight of a term in (13) being defined to be $j$ plus its weight as a term in the inductive expansion. For the verification of (bii) we need one further lemma, whose simple proof we omit.

Lemma 5. Let $T \geqq 0$ be a random variable satisfying the bounds (11), (12). Then for any $s \geqq 1$ there exist $K>0, \varepsilon>0$ such that for all $L \geqq 0, m \geqq 0$, Reg $\geqq 0$,

$$
\sum_{j=0}^{\infty}\left\|e_{L, j}\left(g T_{m}\right)-e_{L, j}\left(g T_{m-1}\right)\right\|_{s} \prec K \tau^{m}\left(1-\varepsilon|g| L^{\gamma}\right)^{-1} .
$$

Now Lemma 1 gives for (13) a majorant

$$
\begin{aligned}
& \exp (|g| E[T]) M\left(1-\varepsilon|g| L^{\gamma}\right)^{-r-1}\|R\|_{v} \\
& \quad+\sum_{m=0}^{\infty} \sum_{j=0}^{\infty}\left\|R\left[e_{L, j}\left(g T_{m}\right)-e_{L, j}\left(g T_{m-1}\right)\right]\right\|_{v} M\left(1-\varepsilon|g| L^{\gamma}\right)^{-\max (r, m)-1}
\end{aligned}
$$


Here $v$ is chosen so $1<v<q$, and we suppose $|g| \leqq 1$. We write $v^{-1}=q^{-1}+s^{-1}$, and use Hölder's inequality and (14) to majorise (15) by

$$
\begin{aligned}
\exp (|g| E[T]) M\left(1-\varepsilon|g| L^{\gamma}\right)^{-r-1}\|R\|_{q} \\
+\|R\|_{q} K M\left(1-\varepsilon|g| L^{\gamma}\right)^{-2} \sum_{m=0}^{\infty} \tau^{m}\left(1-\varepsilon|g| L^{\gamma}\right)^{-\max (r, m)} .
\end{aligned}
$$

(16) may be majorised by the right side of (4) by an appropriate redefinition of $M, \varepsilon$. The proof of Theorem 1 is complete.

\section{Construction of the Polymer Measure for Large Values of the Coupling Constant}

We retain the notation and hypotheses of Theorem [1]. The construction of [1] gives, for sufficiently small positive values of the coupling constant $g, 0 \leqq g<\bar{g}$, a martingale $\left\{f_{m}(g), m \geqq 0\right\}$ relative to the increasing sequence of $\sigma$-algebras $G(m)$, $m \geqq 0$, such that, for some $p>1, K>0, \rho>1$, and all $m \geqq 0$,
(a) $f_{m}(g) \geqq 0$,
(b) $\left\|f_{m}(g)\right\|_{1}=1$,
(c) $\left\|f_{m}(g)\right\|_{p} \leqq K \rho^{m}$.

From (b), (c) we have, by interpolation, for any $t, 0<t<1$,

with

$$
\left\|f_{m}(g)\right\|_{q(t)} \leqq\left\|f_{m}(g)\right\|_{p}^{t} \leqq K^{t} \rho^{m t}
$$

$$
q(t)=p[t+(1-t) p]^{-1}
$$

Now let $N \geqq 1$ be an integer and define probability measures $\alpha(N, g), v(N, g)$, for $0 \leqq g<2^{N / 2} \bar{g}$, by

$$
\begin{aligned}
\alpha(N, g) & =\prod_{v \in T(N)} \zeta(v)_{*}\left[v\left(g 2^{-N / 2}\right)\right] \\
\frac{d \nu(N, g)}{d \alpha(N, g)} & =\frac{1}{Z(N, g)} \exp [-g S(N-1)] .
\end{aligned}
$$

In (2), $\zeta(v)_{*}$ denotes the map induced by $\zeta(v): \Omega \rightarrow \Omega$ on measures; in (3), $Z(N, g)$ denotes the normalisation factor $E_{\alpha(N, g)}[\exp (-g S(N-1))]$. Note that formally

$$
\frac{d \nu(g)}{d \mu}=\frac{1}{Z} \exp \left[-g \sum_{m=0}^{\infty} X(m)\right],
$$

and that, for any $N \geqq 1$,

$$
\mu=\prod_{v \in T(N)} \zeta(v)_{*} \mu
$$

so that, again formally,

$$
\begin{aligned}
\frac{d v(N, g)}{d \mu} & =\frac{1}{Z} \exp [-g S(N-1)] \prod_{v \in T(N)} \zeta(v)^{*}\left[\frac{d v\left(g 2^{-N / 2}\right)}{d \mu}\right] \\
& =\frac{1}{Z} \exp \left[-g S(N-1)-g \sum_{m=0}^{\infty} \sum_{v \in T(N)} 2^{-N / 2} \zeta(v)^{*}[X(m)]\right]
\end{aligned}
$$




$$
\begin{aligned}
& =\frac{1}{Z} \exp \left[-g S(N-1)-g \sum_{m=0}^{\infty} X(m+N)\right] \\
& =\frac{d v(g)}{d \mu} .
\end{aligned}
$$

$v(N, g)$ is thus a candidate for an extension of $v(g)$ to the interval $\left[0,2^{N / 2} \bar{g}\right)$.

The construction of $v(N, g)$ from $\alpha(N, g)$ via (3) requires some comment since the random variable $X$ (and so also $S(N-1)$ ) is not universally defined, but in the first instance only relative to the probability measure $\mu$. In order to define $X$ as a random variable relative to the probability measure $\alpha(N, g)$, we begin with the series

$$
X=\sum_{m \geqq 1}(E[X \mid G(m)]-E[X \mid G(m-1)]) .
$$

The summands in (4) are well-defined relative to $\alpha(N, g)$ since, for each $m \geqq 1$, $\alpha(N, g)|G(m) \ll \mu| G(m)$. Moreover

$$
\begin{aligned}
& E_{\alpha(N, g)}\left[|E[X \mid G(m)]-E[X \mid G(m-1)]|^{2}\right] \\
& \quad=E\left[|E[X \mid G(m)]-E[X \mid G(m-1)]|^{2} \prod_{v \in T(N)} \zeta(v)^{*}\left[f_{m}\left(g 2^{-N / 2}\right)\right]\right] \\
& \quad \leqq\|E[X \mid G(m)]-E[X \mid G(m-1)]\|_{2 r}^{2} \exp \left[t 2^{N} \log \left(K \rho^{m}\right)\right],
\end{aligned}
$$

by Hölder's inequality, and (1). In (5) $r$ denotes the Hölder index conjugate to $q(t)$. We choose $t$ sufficiently small that $\tau=\tau_{2} \exp \left[t 2^{N} \log \rho\right]<1$, with $\tau_{2}$ the constant in (2.1.2) [1], so that (5) is bounded by $C \tau^{m}$, for some $C>0$, and conclude that (4) converges in $L^{2}(\Omega, \alpha(N, g))$. The random variable $X$ defined by (4) is non-negative, since the partial sums $E[X \mid G(m)]$ of (4) are non-negative. In the same way we may define, for any $v \in T, X(v)$ as a non-negative random variable relative to $\alpha(N, g)$, so that the construction of $v(N, g)$ via (3) is well-defined.

To verify that $v(N, g)$ is indeed an extension of $v(g)$, we begin with the case $N=1$. By choosing $\bar{g}$ smaller if necessary, we may arrange that, for any localised and bounded random variable $R$, the expectations $E_{v(g)}[R], E_{v(1, g)}[R]$ are analytic in $g$ for $0 \leqq g<\bar{g}$, and admit asymptotic series as $g \rightarrow 0+$ from which they may be recovered by the Borel summation method. The formal identity of $v(g), v(1, g)$ noted above guarantees the identity of the asymptotic series, and hence of their Borel sums, so that

$$
E_{v(g)}[R]=E_{v(1, g)}[R]
$$

for any localised and bounded $R$; this implies $v(g)=v(1, g)$.

Next for any $N \geqq 1$ and $0 \leqq g<\bar{g} 2^{N / 2}$, we have

$$
\begin{aligned}
\alpha(N, g) & =\prod_{v \in T(N)} \zeta(v)_{*}\left[v\left(g 2^{-N / 2}\right)\right] \\
& =\prod_{v \in T(N)} \zeta(v)_{*}\left[v\left(1, g 2^{-N / 2}\right)\right] \\
& \ll \prod_{v \in T(N)} \zeta(v)_{*}\left[\zeta(0)_{*} v\left(g 2^{-(N+1) / 2}\right) \zeta(1)_{*} v\left(g 2^{-(N+1) / 2}\right)\right] \\
& =\alpha(N+1, g),
\end{aligned}
$$


with

$$
\begin{aligned}
\frac{d \alpha(N, g)}{d \alpha(N+1, g)} & =\frac{1}{\left[Z\left(1, g 2^{-N / 2}\right)\right]^{2^{N}}} \exp \left[-g \sum_{v \in T(N)} 2^{-N / 2} \zeta(v)_{*} X\right] \\
& =\frac{1}{\left[Z\left(1, g 2^{-N / 2}\right)\right]^{2 N}} \exp [-g X(N)],
\end{aligned}
$$

so that $v(N+1, g)$ and $v(N, g)$ are both absolutely continuous with respect to $\alpha(N+1, g)$; the identity $v(N+1, g)=v(N, g)$ is then established by checking that

$$
\frac{d v(N+1, g)}{d \alpha(N+1, g)}=\frac{d v(N, g)}{d \alpha(N+1, g)} .
$$

We may now write $v(N, g)=v(g)$, so that $v(g)$ is defined for all $g \geqq 0$. In 3.2 [1] we have shown that, for sufficiently small $g$, the measure $v(g)$ induces a measure on the space of continuous paths $\mathbf{x}:[0,1] \rightarrow \mathbb{R}^{3}$ with $\mathbf{x}(0)=\mathbf{0}$ via the Ciesielski map 3.2.5 [1]. Since the above construction of $v(g)$ for large values of $g$ gives $v(g) \ll \alpha(N, g)$ for any $N \geqq 1$, and since the product measure $\alpha(N, g)$ induces a measure on the space of continuous paths for all sufficiently large $N$ by the result of 3.2 [1], it follows that $v(g)$ induces a measure on the space of continuous paths for all $g \geqq 0$.

We have seen in Sect. 2 that, for sufficiently small $g, v(g)$ is analytic in $g$ in the sense that, for any bounded localised random variable $R, E_{v(g)}[R]$ is analytic in $g, 0<g<\bar{g}$, and is uniquely determined by its asymptotic expansion as $g \rightarrow 0+$, i.e. by the perturbation series. The following Theorem 2 completes this result by showing that $E_{v(g)}[R]$ is analytic in $g$ for all $g>0$. This rules out, as expected, any "phase transition" for a finite value of $g$, and establishes the uniqueness of $v(g)$ in the strongest possible sense.

Theorem 2. There exists a constant $D>0$ such that, for any bounded localised random variable $R, E_{v(g)}[R]$ admits a meromorphic extension to the open set $U=\left\{g: \operatorname{Reg}>0,|\operatorname{Im} g|<D(1+\operatorname{Reg})^{-1}\right\}$ with poles (if any) independent of $R$.

Proof. Fix a truncation sequence $B$ as in Sect. 2 , and denote by $v(g ; B)$ the measure constructed using the truncated random variables $X(n ; B)$, and by $T$ the tail of the interaction energy, given by $(2.9)$, so that $v(g) \ll v(g ; B)$, with

$$
\frac{d v(g)}{d v(g ; B)}=\frac{\exp (-g T)}{E_{v(g ; B)}[\exp (-g T)]}
$$

For any integer $N \geqq 1$ define the measure

$$
\alpha(N, g ; B)=\prod_{v \in T(N)} \zeta(v)_{*}\left[v\left(g 2^{-N / 2} ; B\right)\right],
$$

and the positive random variables

$$
\begin{aligned}
T_{N} & =2^{-N / 2} \sum_{v \in T(N)} \zeta(v)^{*}[T], \\
K_{N} & =S(N-1)+T_{N} .
\end{aligned}
$$

Then from (3), (6), and the fact that $v(N, g)=v(g)$, it follows that $v(g) \ll \alpha(N, g ; B)$, 
with

$$
\frac{d v(g)}{d \alpha(N, g ; B)}=\frac{\exp \left(-g K_{N}\right)}{E_{\alpha(N, g ; B)}\left[\exp \left(-g K_{N}\right)\right]} .
$$

Fix $q>1$. From the proof of Theorem 1 [1] and the additional remarks on the extension to complex values of $g$ in Sect. 2, we have the following information concerning the measures $v(g ; B)$ : There exists $g_{0}>0, C>0$ such that for $0 \leqq g \leqq g_{0}$, and any $m \geqq 0, v(g ; B) \mid G(m) \ll \mu$, with a Radon-Nikodym derivative $f_{m}(g ; B) \in L^{q}(\Omega, \mu)$ which admits a holomorphic extension (as a function of $g$ with values in $L^{q}$ ) to $\left\{g:|g| \leqq g_{0}\right\}$ satisfying

$$
\left\|f_{m}(g ; B)\right\|_{q} \leqq C^{m}
$$

Note also that $\left\|f_{m}(g ; B)\right\|_{1}=1$ for $0 \leqq g \leqq g_{0}$.

Now choose $g_{1}, 0<g_{1}<\frac{1}{2} g_{0}$, and consider the functions $h_{m}(\theta)=f_{m}\left(g_{1}[1+\right.$ $\cosh (\theta)])$. They are holomorphic in the strip $0 \leqq \operatorname{Re} \theta \leqq \theta_{0}\left(\theta_{0}=\operatorname{ch}^{-1}\left(g_{0} g_{1}^{-1}-1\right)\right)$ with values in $L^{q}(\Omega, \mu)$, and satisfy

$$
\begin{array}{ll}
\left\|h_{m}(\theta)\right\|_{q} \leqq C^{m}, & \operatorname{Re} \theta=\theta_{0} \\
\left\|h_{m}(\theta)\right\|_{1}=1, & \operatorname{Re} \theta=0 .
\end{array}
$$

By complex interpolation it follows that, for any $t, 0 \leqq t \leqq 1$,

$$
\left\|h_{m}(\theta)\right\|_{q(t)} \leqq C^{m t},
$$

for $\theta$ in the strip $0 \leqq \operatorname{Re} \theta \leqq t \theta_{0}$. Here $q(t)$ is given by

(12) is equivalent to

$$
q(t)^{-1}=t^{-1}+(1-t) q^{-1} \text {. }
$$

$$
\left\|f_{m}(g ; B)\right\|_{q(t)} \leqq C^{m t},
$$

for $g$ in the closed region $K(t)$ bounded by the ellipse $E(t)$ with foci at $0,2 g_{1}$ and minor axis $g_{1} \sinh \left(t \theta_{0}\right)$. We denote by $I(t)$ the interior of $K(t)$.

The representation (10) of $v(g)$ gives a representation of $E_{v(g)}[R]$ as a quotient

$$
E_{v(g)}[R]=\frac{E_{\alpha(N, g ; B)}\left[R \exp \left(-g K_{N}\right)\right]}{E_{\alpha(N, g ; B)}\left[\exp \left(-g K_{N}\right)\right]} .
$$

We will show that the numerator and denominator of (14) are holomorphic in a certain open set $V_{N}$, independent of $R$, so that $E_{v(g)}[R]$ is meromorphic in

$$
V=\bigcup_{N \geqq 1} V_{N} \text {. }
$$

We will then show that $V$ contains the set $U=U(D)$ of the theorem statement for some $D \geqq 0$, and the proof of Theorem 2 will be complete.

Evidently it suffices to consider (for arbitrary $R$ ) the numerator of (14), the denominator being obtained as a special case for $R=1$. Recall that $R$ is localised, i.e. $R \in G(r)$ for some $r \geqq 0$. We consider the expansion

$$
\begin{aligned}
E_{\alpha(N, g ; B)}\left[R \exp \left(-g K_{N}\right)\right]= & E\left[R \exp \left(-g E\left[K_{N} \mid G(r)\right]\right) f_{r}(N, g ; B)\right] \\
& +\sum_{s=r}^{\infty} E\left[R \left\{\exp \left(-g E\left[K_{N} \mid G(s+1)\right]\right)\right.\right. \\
& \left.\left.-\exp \left(-g E\left[K_{N} \mid G(s)\right]\right)\right\} f_{s+1}(N, g ; B)\right],
\end{aligned}
$$


with

$$
f_{m}(N, g ; B)=\frac{d(\alpha(N, g ; B) \mid G(m))}{d \mu}=E\left[\prod_{v \in T(N)} \zeta(v)^{*}\left[f_{m}\left(g 2^{-N / 2} ; B\right)\right] \mid G(m)\right] .
$$

Our earlier estimates give for some $K>0$, and all $N \geqq 1, p \geqq 1, m \geqq 0$ a bound

$$
\left\|K_{N}-E\left[K_{N} \mid G(m)\right]\right\|_{p} \leqq K(N+1) p^{1+\gamma} \tau^{m} .
$$

In (18) $\tau<1$, and $\gamma$ is as in Sect. 2 (cf. Lemma 4). Now set $t_{N}=t_{0} 2^{-N}$ with $t_{0}>0$ sufficiently small that

$$
\tau C^{t_{0}}<1 .
$$

Thus using Hölder's inequality with index $q\left(t_{N}\right)$, the fact that $K_{N} \geqq 0$, and (13), (17), (18), (19) it is easy to check that the terms of (16) are holomorphic in

$$
V_{N}=\left\{g: \operatorname{Reg}>0,2^{-N / 2} g \in I\left(t_{N}\right)\right\},
$$

and that the series converges uniformly on $V_{N}$, so that its sum $E_{\alpha(N, g ; B)}$ $\left[R \exp \left(-g K_{N}\right)\right]$ is holomorphic in $V_{N}$.

Set $V=\bigcup_{N \geqq 1} V_{N}$. Given $g \geqq g_{1}$ we may choose $N$ so $\frac{1}{2} g_{1} \leqq 2^{-N / 2} g \leqq g_{1}$. Then $2^{-N / 2} g \in I\left(t_{N}\right)$, and its distance from $\partial I\left(t_{N}\right)=E\left(t_{N}\right)$ is $\geqq C t_{N}$ for some $C>0$. Thus the distance from $g$ to $\partial V$ is at least $C 2^{N / 2} t_{N}=C t_{0} 2^{-N / 2} \geqq C_{1} g^{-1}$, with $C_{1}=C t_{0} g_{1} 2^{-1}$. For some $\varepsilon>0, V_{1}$ contains $\left\{g: 0<\operatorname{Reg}<g_{1},|\operatorname{Im} g|<\varepsilon\right\}$. Hence $V$ contains $U(D)$ for $D=\min \left\{\varepsilon, C_{1}\right\}$.

Remark. By using Jensen's inequality to obtain a crude lower bound for the denominator of (14), we can determine explicitly an open set $W$ containing the positive real axis in which $E_{v(g)}[R]$ is holomorphic for any bounded, localised $R$. We find for some $A, \mathrm{C}>0$

$$
W=\left\{g: \operatorname{Reg}>0,|\operatorname{Im} g|<A \exp \left(-C(\operatorname{Reg})^{2+2 \beta}\right)\right\} .
$$

\section{Disjointness of the Measures $v(g)$}

We adopt the notation of 3.1 [1]. Let $\lambda_{1}, \lambda_{2}$ be positive measures satisfying 3.1 .10 [1], so that the random variables $J\left(\lambda_{1}\right), J\left(\lambda_{2}\right)$ are well-defined, and have finite variance. Then

$$
\begin{aligned}
E\left[J\left(\lambda_{1}\right) J\left(\lambda_{2}\right)\right] & =\int \frac{d \lambda_{1}\left(\xi_{1}\right) d \lambda_{2}\left(\xi_{2}\right)}{\left[C\left(\xi_{1}, \xi_{2}\right) C\left(\xi_{2}, \xi_{2}\right)-C\left(\xi_{1}, \xi_{2}\right)^{2}\right]^{v / 2}} \\
& \geqq \int \frac{d \lambda_{1}\left(\xi_{1}\right)}{\left[C\left(\xi_{1}, \xi_{1}\right)\right]^{v / 2}} \frac{d \lambda_{2}\left(\xi_{2}\right)}{\left[C\left(\xi_{2}, \xi_{2}\right)\right]^{v / 2}} \\
& =E\left[J\left(\lambda_{1}\right)\right] E\left[J\left(\lambda_{2}\right)\right] .
\end{aligned}
$$

Thus the random variables $X(v)$ which appear in the construction of the polymer measure $(3.2[1])$ satisfy the following condition:

$C 7$. For any $v_{1}, v_{2} \in T, X\left(v_{1}\right)$ and $X\left(v_{2}\right)$ are positively correlated.

The purpose of this section is to show that the polymer measures for different 
values of the coupling constant $g$ are pairwise disjoint. This result follows from the above remark and Theorem 3 below.

Theorem 3. Retain the notation and hypothesis of Theorem 1 [1], and suppose also that $C 7$ holds. Then for any $g_{1}, g_{2} \geqq 0$, with $g_{1} \neq g_{2}$, the measures $v\left(g_{1}\right), v\left(g_{2}\right)$ are disjoint.

Proof. Note first that if, for some $N \geqq 1, v\left(2^{-N} g_{1}\right)$ and $v\left(2^{-N} g_{2}\right)$ are disjoint, then the product measures $\alpha\left(N, g_{1}\right), \alpha\left(N, g_{2}\right)$ of Sect. 2 are disjoint, and hence $v\left(g_{1}\right) \ll \alpha\left(N, g_{1}\right)$ and $v\left(g_{2}\right) \ll \alpha\left(N, g_{2}\right)$ are disjoint. It therefore suffices to prove Theorem 3 for $g_{1}, g_{2} \in[0, \bar{g})$, with $\bar{g}$ the constant appearing in Theorem 1 [1].

Next notice that the proof given in [1] that $S(n)$ is approximately Gaussian for large $n$ remains valid if $g S(n)$ is replaced by

$$
\widetilde{S}(n)=\sum_{m=0}^{n} g(m) X(m)
$$

with $\{g(m), m \geqq 0\}$ an arbitrary sequence of real numbers chosen from $[0, \bar{g})$. More precisely, we obtain, by the expansion and majorisation method of [1], for $\tilde{Z}_{n}=E[\exp (-\tilde{S}(n))]$ upper and lower bounds

$$
K_{1} \widetilde{C}_{n} \leqq \widetilde{Z}_{n} \leqq K_{2} \widetilde{C}_{n}
$$

uniform in $n$, and in the sequence $\{g(m), m \geqq 0\}$. (In (1), $K_{1}, K_{2}$ are constants $>0$, and $\widetilde{C}_{n}=\exp \left\{-E[\tilde{S}(n)]+\frac{1}{2} \operatorname{Var}[\tilde{S}(n)]\right\}$ is the value which $\tilde{Z}_{n}$ would have if $\tilde{S}(n)$ were Gaussian.) that

To show $v\left(g_{1}\right), v\left(g_{2}\right)$ disjoint it suffices to find, for any given $\varepsilon>0$, a set $E$ such

We will show that

$$
v\left(g_{1}\right)(E) \leqq \varepsilon, \quad v\left(g_{2}\right)\left(E^{c}\right) \leqq \varepsilon .
$$

$$
E=\{\omega: S(k) \geqq \eta\}
$$

is such a set, if the integer $k$ and real number $\eta$ are properly chosen.

For some $C>0$, and all integers $n, k$ with $n \geqq k$ we have

$$
k \operatorname{Var}[X] \leqq \operatorname{Var}[S(k)] \leqq \operatorname{Cov}[S(n) S(k)] \leqq C k,
$$

$C 7$ being used to obtain the first two inequalities. Hence

$$
H(k)=\lim _{n \rightarrow \infty} \operatorname{Cov}[S(n) S(k)]
$$

exists as the limit of a bounded increasing sequence, and $H(k) \rightarrow \infty$ as $k \rightarrow \infty$.

For definiteness suppose $g_{1}>g_{2}$, and choose $\beta$ so $0<\beta<g_{1}-g_{2}$. For any integer $n$ we have

$$
\begin{gathered}
v_{n}\left(g_{1}\right)(E) \leqq \int \exp [\beta(S(k)-\eta)] d v_{n}\left(g_{1}\right), \\
v_{n}\left(g_{2}\right)\left(E^{c}\right) \leqq \int \exp [-\beta(S(k)-\eta)] d v_{n}\left(g_{2}\right)
\end{gathered}
$$

Using the definition 2.1.4 [1] of $v_{n}$, the right side of (4) may be written in the form

$$
\exp (-\beta \eta) \tilde{Z}_{n}(A) \tilde{Z}_{n}^{-1}(B)
$$


with $A, B=\{g(m), m \geqq 0\}$ the sequences given by

$$
\begin{aligned}
A: g(m) & =g_{1}-\beta, \quad 0 \leqq m \leqq k \\
g(m) & =g_{1}, \quad m>k \\
B: g(m) & =g_{1} \quad \text { for all } m
\end{aligned}
$$

We may therefore use (1) to obtain an upper bound for the right side of (4). With the choice of $\eta$ fixed by

$$
\eta=E[S(k)]-\frac{1}{2}\left(g_{1}+g_{2}\right) H(k),
$$

and the help of the middle inequality of (3), this leads in the limit $n \rightarrow \infty$ to

$$
v\left(g_{1}\right)(E) \leqq K_{2} K_{1}^{-1} \exp \left[-\frac{1}{2} \beta\left(g_{1}-g_{2}-\beta\right) H(k)\right] .
$$

Proceeding in the same way from (5) we find that the right side of (7) is also an upper bound for $v\left(g_{2}\right)\left(E^{c}\right)$. Since $H(k) \rightarrow \infty$ as $k \rightarrow \infty$, it remains only to choose $k$ sufficiently large to obtain (2), and hence to complete the proof of Theorem 3.

Acknowledgement. The author wishes to thank David Ragozin for a valuable discussion in connection with the proof of Theorem 2 .

\section{References}

1. Westwater, M. J.: On Edwards' model for long polymer chains. Commun. Math. Phys. 72, 131-174 (1980)

2. Edwards, S. F.: The statistical mechanics of polymers with excluded volume. Proc. Phys. Soc. 85, 613624 (1965)

3. Westwater, M. J.: On Edwards' model for polymer chains. II The self-consistent potential. Commun. Math. Phys. 79, 53-73 (1981)

4. Symanzik, K.: Euclidean quantum field theory. In: Local quantum theory, Jost, R. (ed.) New York: Academic Press 1969

5. Sokal, A. D.: An improvement of Watson's theorem on Borel summability. J. Math. Phys. 21, 261-265 (1980)

Communicated by E. Lieb

Received August 24, 1981 